\title{
ALIMENTATION DES OVINS DES MARCHES DE VENTE DE BETAIL DANS LA VILLE D'ABIDJAN, CÔTE D'IVOIRE
}

\author{
F. A. KOUASSI' ${ }^{1}$ V. MAJOREIN²; J.IPOU IPOU' ; C.Y. ADOUYAO' ${ }^{1}$ tK. KAMANZI ${ }^{1}$ \\ 1Université de Cocody-Abidjan, UFR Biosciences Laboratoire de Botanique, 22 B.P. 582 Abidjan 22, Côte d'Ivoire. \\ E-mail : akossouafifi@yahoo.fr \\ ${ }^{2}$ Université libre de Bruxelles, Service d'Ecologie du Paysage et Systèmes de production végétale, Avenue F. D. \\ Roosevelt 50 - CP169, B-1050 Bruxelles, Belgique.
}

\begin{abstract}
RESUME
Une étude a été menée pour déterminer l'alimentation, les lieux et contraintes d'approvisionnement des aliments des ovins présents sur les marchés à bétail dans la ville d'Abidjan. Elle a été réalisée à partir d'enquêtes et d'inventaires floristiques effectués sur les marchés à bétail. II ressort de cette étude que les fourrages constitués, par ordre d'importance, des espèces telles que : Panicum maximum Jacq. (Poaceae), Chloris pilosa Schumach. (Poaceae), Brachiaria lata (Schumach.) Hub (Poaceae); Mariscus cylindristachyus Steud. (Cyperaceae), Cyperus longibracteatus (Cherm.) Kuk (Cyperaceae) sont les plus consommées par les animaux. Les lieux de collecte de ces fourrages sont les jachères, les abords de routes et les bas fonds de la ville. Certaines contraintes telles que : (i) manque de liquidité financière, (ii) retard dans les livraisons des fourrages, (iii) absence de structures adaptées à cette activité, (iiii) manque de moyens adéquats pour le prélèvement des fourrages rendent difficile le bon déroulement de l'approvisionnement de ces fourrages. Enfin le développement urbain, commande que l'on mette en place des structures spécialisées dans l'alimentation des ovins et identifie des lieux appropriés à l'approvisionnement en ces fourrages.
\end{abstract}

Mots clés : Marché, ovins, fourrages, Côte d'Ivoire.

\author{
ABSTRACT \\ FEEDING OF OVINE SOLD IN THE LIVESTOCK MARKET OF ABIDJAN, CÖTE D'IVOIRE
}

A study was conducted on the livestock in order to asses feeding, places and constraints of food supplied to the ovine sold at market in Abidjan. It was carried out through investigations and floristic inventories. Data analysis showed that the following plant species: Panicum maximum Jacq. (Poaceae), Chloris pilosa Schumach. (Poaceae), Brachiaria lata (Schumach.) Hub (Poaceae); Mariscus cylindristachyus Steud. (Cyperaceae), Cyperus longibracteatus (Cherm.) Kuk (Cyperaceae) were the most consumed by the animals. Forage collection areas, fallows lowland areas around the city of Abidjan, as well as along roadsides. Constraints related to fodder supplying were (i) funds availability, (ii) delay in fodder deliveries, (iii) lack of adequate infrastructures in livestock feeding, as well as identify appropriate forage collection sites.

Key words : Market, ovine, fodder, Côte d'Ivoire. 


\section{INTRODUCTION}

La Côte d'Ivoire est déficitaire en produits animaux. En effet, en dépit des efforts entrepris par l'Etat Ivoirien, la couverture des besoins en protéines animales de la population reste, largement tributaire des marchés extérieurs (Anonyme, 1999 ; Koffi-Koumi et al., 2001 ). La productivité du cheptel demeure encore faible et le pays importe près de la moitié de ses besoins en viande. L'approvisionnement en bétail de l'extérieur place la Côte d'Ivoire au rang d'un des plus grands importateurs de ruminants (ovins et bovins) en Afrique de l'Ouest (Anonyme, 2001). Les animaux, en particulier les ovins, importés des pays limitrophes (Burkina, Mali, Niger) arrivent par voies ferrées et/ou routières. Ils transitent généralement par le principal marché de bétail d'Abidjan (Port-Bouet) avant d'être redistribués sur les sites secondaires de commercialisation des autres quartiers (Attécoubé, Abobo, Adjamé, etc.). Les mauvaises conditions d'importation (durée du trajet, entassement dans les véhicules et/ou wagon, mauvaise alimentation, parcage au lieu de transit, etc.) occasionnent souvent la fatigue, la perte de poids, voire des maladies chez certains animaux. Pour rentabiliser leur commerce, les éleveurs et autres intermédiaires soumettent d'abord les animaux à une embouche préalable. Une forme d'élevage, à court terme liée, à la présence plus ou moins prolongée des animaux sur les marchés, est pratiquée, avec les mêmes exigences sanitaires et alimentaires que celles des élevages ordinaires (l'élevage de prairies, en enclos et en transhumance). Une bonne alimentation de ces ovins est donc indispensable pour assurer une meilleure vente. En effet, l'alimentation constitue un facteur important qui conditionne la production, le poids et le prix de vente des animaux (Vivier et al., 1975 ; Rivière, 1991). Pour ces mêmes auteurs, les effets d'une carence alimentaire peuvent se noter aussi bien sur la quantité que sur la qualité des produits animaux. Les aliments réservés aux ovins sur les marchés de vente de bétail, sont d'origine végétale ou industrielle, mais aussi proviennent des restes d'aliments, etc.).

Aujourd'hui, selon nos enquêtes, la fourniture des marchés en aliments de bétail est assurée par des marchands occasionnels et le secteur reste encore informel et mal organisé. Les livreurs de fourrages, tous des particuliers, récoltent les fourrages dans plusieurs quartiers de la ville pour ensuite les commercialiser aux vendeurs de bétail. Peu de données fiables, pouvant servir d'outils d'aide à son organisation sont inexis-tantes. Cette étude, la première d'une série, vise d'abord à déterminer la composition alimentaire de ces animaux, afin de déterminer le mode d'alimentation privilégiée pour une meilleure valorisation des produits alimentaires de ces animaux.

\section{MATERIEL ET METHODES}

La méthodologie adoptée a été celle des enquêtes semi structurées, associées à des relevés floristiques. Les enquêtes ont permis d'identifier le mode principal d'alimentation, et de recenser les lieux et les problèmes liés à l'approvisionnement. Les relevés floristiques ont été réalisés dans les mangeoires en vue d'établir la liste floristique des fourrages et en identifier les espèces majeures.

\section{LOCALISATION DES SITES ETUDIES}

Le marché à bétail de Port Bouet le plus grand, est situé dans la zone Sud de la ville, proche de la lagune Ebrié. C'est dans ce marché que sont reçus et parqués les animaux avant redistribution. Les deux marchés d'Atté-coubé, commune située dans le Centre Ouest de la ville, sont plus accessibles à cause de leur proximité au quartier commercial d'Adjamé. Enfin le marché d'Abobo, situé au nord de la ville, est plus accessible aux populations de cette zone. Pour faciliter la gestion des données, les codes M1 et M2 ont été attribués aux deux marchés d'Attécoubé, et M3 et M4 à ceux de Abobo et de Port Bouet, respectivement.

\section{REALISATION DES ENQUETES}

Les enquêtes ont été réalisées dans les 4 principaux marchés de bétail de la ville d'Abidjan dans la période d'août à décembre 2006.

Au total plus de 200 vendeurs d'ovins ont été interrogés, soit 50 vendeurs par marché. Des enquêtes ont aussi été réalisées auprès de 150 livreurs de fourrages sur les marchés de vente de bétails et sur les lieux de prélèvement. Les informations recueillies ont porté sur des fiches d'enquête et ont concerné, entre autres, la composition alimentaire, les espèces fourragères les plus consommées, les techniques et les contraintes liées à l'alimentation des ovins, ainsi que les lieux d'approvisionnement de ces fourrages et le revenu financier des vendeurs de fourrage. Concernant ce dernier cas, les revenus 
issus de la vente des fourrages ont été ceux déclarés par l'exploitant lui-même. L'activité vente de fourrage exige des dépenses qui ne sont pas directement liées à la récolte de fourrage. Ces dépenses concernent les frais annexes lors de la transaction et qui méritent d'être prises en compte dans le cadre d'une analyse financière de la rentabilité après l'opération de vente.

Les différents sites d'approvisionnement ont été visités et recensés avec leurs coordonnées géographiques, à l'aide d'un GPS (Carmin $12 \mathrm{XL})$.

Le test de Chi carré a été réalisé à l'aide du logiciel Statistica en se servant des résultats des tableaux croisés dynamiques offrant les proportions des types d'alimentation et celles des lieux de prélèvement. De même, le logiciel Arc View Gis 3.3 a été utilisé pour dresser la carte de répartition des différents sites de collectes de fourrages. Le revenu financier a été calculé à l'aide du tableur excel.

\section{INVENTAIRE FLORISTIQUE}

Au total, 50 mangeoires par marché, soit 200 mangeoires ont été choisies de façon aléatoire. Un inventaire floristique, qui a consisté à dresser la liste des plantes présentes dans chaque mangeoire a été réalisé. Les espèces identifiées ont été affectées d'un coefficient d'abondance selon l'échelle suivante : 1 pour les espèces non abondantes, avec moins de $10 \%$ de présence, 2 pour les espèces peu abondantes (entre 10 et $30 \%$ de présence), 3 pour les espèces abondantes (entre 30 et $50 \%$ de présence) et 4 espèces très abondantes (entre 50 et $100 \%$ de présence).

\section{APPETIBILITE DES ESPECES}

La valeur fourragère tient compte, en général, de l'appétibilité, la valeur nutritive et la productivité (abondance) des espèces (Daget et Poissonnet, 1990). Dans notre étude, la valeur fourragère des espèces a été évaluée à partir de l'appétibilité et de l'abondance dans les mangeoires. L'appétibilité désigne les caractéristiques de l'aliment qui provoquent une réaction des sens de l'animal. Elle est le corollaire de l'appétit de l'animal pour un aliment donné (Church, 1979 ; Matthews, 1983). Les vendeurs d'ovins offrent les espèces fourragères les plus appréciées par les ovins. Ainsi, l'espèce la plus abondante dans la mangeoire constitue le fourrage le mieux consommé, le plus prisé par les animaux et donc de bonne valeur fourragère. Ainsi, sur la base des espèces abondantes dans les mangeoires, et par comparaison à la classification de la valeur fourragère des espèces définies dans les pâturages sahéliens (Ould Soulé, 1995), les espèces recensées ont été groupées en 4 classes selon la valeur fourragère :

- classe 4 : espèces très appétées (TA), ingérées plus rapidement et plus abondantes ;

- classe 3 : espèces moyennement appétées (MA), moins ingérées et moins abondantes ;

- classe 2 : espèces peu appétées (PA), ingérées lentement, et moins abondantes ;

- classe 1 : espèces non appétées (NA), non ingérées, espèces intruses, épineuses ou toxiques.

\section{RESULTATS}

\section{COMPOSITION DE L'ALIMENTATION DES OVINS SUR LES MARCHES DE VENTE}

Sur les 4 principaux marchés à bétail de la ville d'Abidjan, l'alimentation des ovins est composée en moyenne de $90 \%$ de fourrages verts et de $10 \%$ de compléments alimentaires. Ces derniers sont constitués de sous-produits achetés et distribués aux ovins pendant les heures d'attente du fourrage. Parmi eux, les sons de céréales (mil, riz, maïs, sorgho), provenant des moulins et des ménages ; les épluchures de banane, d'igname et surtout de manioc sont les plus utilisés car plus disponibles. Le sel est apporté sous forme dissoute dans l'eau.

\section{MODE D'ALIMENTATION ET CONTRAINTES}

Les enquêtes et observations ont montré que les vendeurs d'ovins nourrissent leur bétail à travers la distribution d'aliments dans les mangeoires à toutes les périodes de l'année. Ce mode d'alimentation se fait de deux manières : l'affouragement en vert et l'affouragement en vert associé aux sous-produits. Sur 200 personnes enquêtées, les résultats ont montré que $76,5 \%$ apportent un complément alimentaire en plus des fourrages verts, et $23,5 \%$ ne servent que des fourrages aux ovins. 
Les fourrages sont achetés aux vendeurs par bottes de 1,5 à $3 \mathrm{~kg}$ ou de 4 à $6 \mathrm{~kg}$ (Figure 1) à des prix variant entre 50 et 200F CFA, suivant le poids et la période de vente. La quantité d'herbe donnée aux ovins est en moyenne de 2 à 4 bottes par jour et par tête selon le poids de l'animal et la botte de fourrage. Ainsi, les ovins de petits poids consomment, en moyenne, 2 bottes par jour, et les plus gros, 4 bottes.

Plusieurs contraintes liées à ce mode d'alimentation des ovins sont à signaler. Elles s'observent aussi bien chez les acheteurs (vendeurs de bétail) que chez les vendeurs d'herbage. Chez les acheteurs, le manque de liquidité financière pour l'achat des fourrages pendant certaines périodes de l'année, les emmène à contracter un crédit chez les vendeurs d'herbage, à diminuer la ration alimentaire des ovins et aussi à s'orienter vers les sous-produits. Chez les vendeurs d'herbages, le retard dans les livraisons des fourrages entraîne un manque à gagner : l'inorganisation de cette activité due à l'absence de structures adaptées ; les moyens de prélèvement et d'acheminement des fourrages vers les marchés restent rudimentaires. Cela constituent la principale cause des retards dans les livraisons. L'absence de méthode de conservation des fourrages entraîne aussi leur fanaison et une perte financière en cas de difficultés d'écoulement.

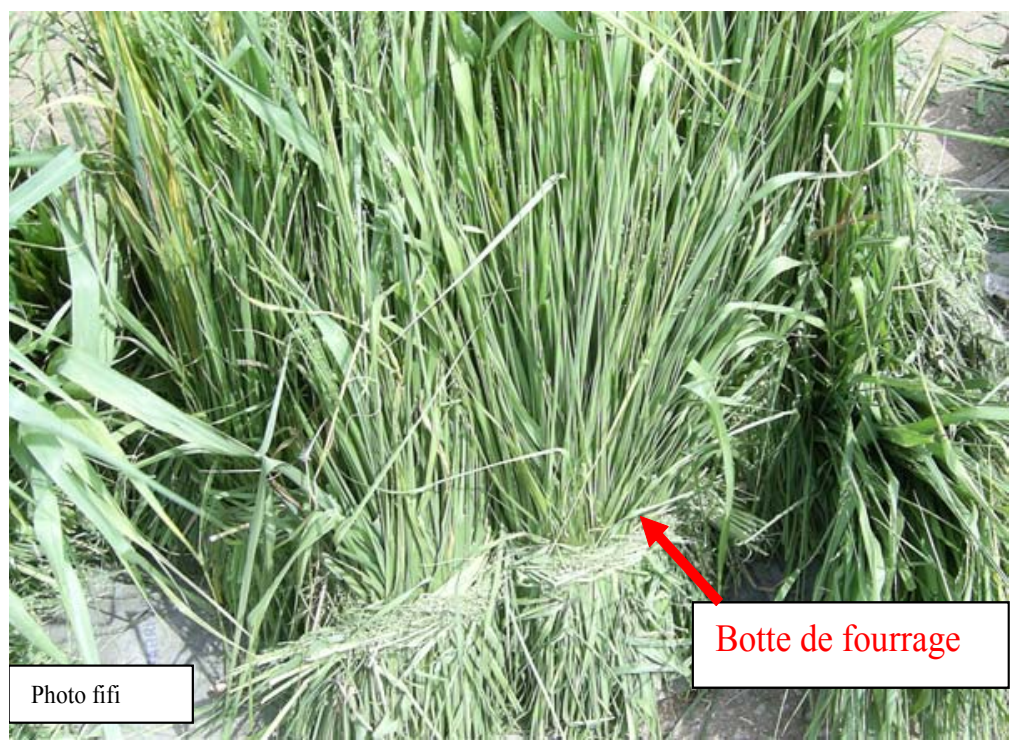

Figure 1 : Bottes de fourrage prêtes à être acheminées vers un marché de bétail.

Bundle of fodder ready to be forward to the livestock market.

\section{LIEUX ET MODE DE PRELEVEMENT DES FOURRAGES}

Le prélèvement des fourrages se fait sur des jachères dans des végétations spontanées, des bas-fonds, et sur des espaces verts aménagés, mais non entretenus, autour de constructions inachevées, sur des terres incultes, et aux abords des routes dans plusieurs quartiers de la ville d'Abidjan. Le test de Chi-deux ( $x^{2}$ : $44,0829 ; d d l=16 ; P=0,0001916)$ révèle des sites de prélèvement privilégiés. Les jachères, les bas-fonds et les abords des routes sont les plus exploités. Sur la population enquêtée,
$28 \%$ privilégie la récolte des fourrages dans les jachères, et les bas-fonds, et 26,67\% dans les abords des routes. Le prélèvement se fait de façon mécanique à l'aide de couteaux ou de faucilles. L'achemi-nement vers les marchés se fait à l'aide de charrettes, de bicyclettes, à pieds et en voiture. La distance entre ces lieux de récolte et les marchés de vente diffère d'un exploitant à un autre et, est fonction des lieux de coupe (Figure 2). Ces exploitant parcourent en moyenne une distance de $15 \mathrm{~km}$ par jour pour récolter leurs produits. La distance minimale parcourue est d'environ $2 \mathrm{~km}$, et le maximl est de $40 \mathrm{~km}$ en fonction des lieux de 
coupes, des périodes d'abondance des ovins sur le marché. Toute chose qui favorisent une hausse de la demande de fourrages.

\section{ESTIMATION DU REVENU FINANCIER JOURNALIER MOYEN D'UN EXPLOITANT}

Le revenu financier journalier moyen varie en fonction des périodes où les marchés sont très ou peu fournis en animaux (Tableau 1). Les enquêtes montrent que le fourrage vendu par les exploitants est prélevé gratuitement dans les différents milieux. L'opération vente de fourrages est entièrement préfinancé par l'exploitant lui- même. Cependant, il faut noter que pour les exploitants enquêtés, le coût du matériel utilisé avait déjà été amorti, si bien que dans l'estimation du revenu financier, il n'a pas été pris en compte. Le prix unitaire (PU) d'une botte est en moyenne de 50 FCFA lorsqu'il ya peu d'animaux sur le marché et de 100 FCFA en cas de forte affluence. Ainsi, nous avons estimé le revenu financier moyen par jour d'un exploitant pendant les périodes de faible affluences d'animaux sur les marchés, à 875 F CFA et 3350 FCFA lorsque les marchés sont très fournis en animaux.

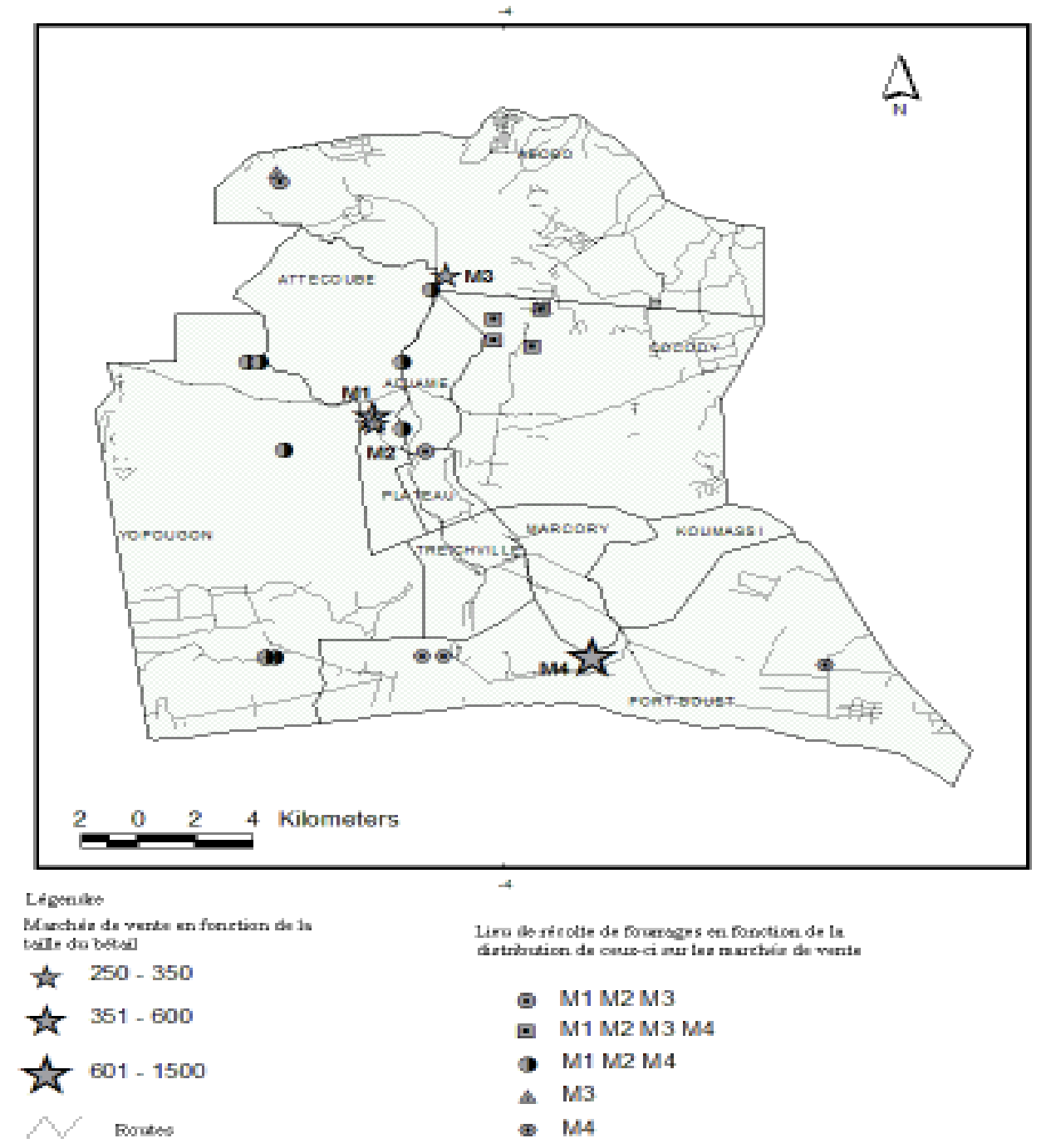

Figure 2 : Marchés de vente de bétail et sites de prélèvement des plantes fourragères dans la ville d'Abidjan (Côte d'Ivoire).

Livestock market and fodder sampling sites in the city of Abidjan (Côte d'lvoire).

M1 = Plus grand marché de bétail de Attécoubé, M2 = Plus petit marché de bétail de Attécoubé, M3 = Marché d'abobo et M4 = Marché de Port Bouet.) 
Tableau 1 : Revenu financier journalier moyen en CFA d'un exploitant de fourrage dans la ville d'Abidjan. Average daily monetary gain (CFA) for a livestock fodder collector in the city of Abidjan.

\begin{tabular}{lccc}
\hline Variable & $\begin{array}{c}\text { Pénurie d'animaux } \\
\text { sur le marché }\end{array}$ & $\begin{array}{c}\text { Abondance d'animaux } \\
\text { sur le marché }\end{array}$ & Total \\
\hline Nombre d'exploitants interrogés & 150 & 150 & 150 \\
Coût d'acquisition de la botte en CFA & 0 & 0 & 0 \\
Nombre moyen de bottes vendues & $(27,5)$ & Compris entre [20-35] & Compris entre [33-54] \\
PU (en CFA) moyen par botte & 50 & 100 & 1000 \\
Charge annexe (CA) en moyenne par jour & 500 & 4350 & 150 \\
PV (en CFA) des n bottes vendues (NBV*PU) & 1375 & 3350 & 5725 \\
RF (en CFA)/jour d'un exploitant (PV-CA) & 875 & 502500 & 4225 \\
RG des exploitants interrogés en une journée de travail & 131250 & 633750 \\
\hline
\end{tabular}

CA : Charge Annexe ; Ca : Coût d'Acquisition ; FCFA : Franc de la Communauté Financière Africaine ; NBV : Nombre de Bottes Vendues ; PU : Prix Unitaire ; PV : Prix de Vente ; RG : Revenu général.

\section{FLORE FOURRAGERE DES MARCHES A BETAIL DE LA VILLE D'ABIDJAN}

La flore fourragère des marchés à bétail de la ville d'Abidjan comprend 97 espèces réparties en 77 genres appartenant à 29 familles. Les principales espèces rencontrées sont les Poaceae fourragères représentées surtout par les espèces Chloris pilosa Schumach. (Poaceae), Panicum maximum Jacq.(Poaceae), Brachiaria lata (Schumach.) Hub (Poaceae), les légumineuses fourragères représentées par Arachis hypogea L. (Fabaceae), Phaseolus vulgaris $L$ (Fabaceae). En plus de ces deux groupes d'espèces végétales, certaines espèces telles que Commelina erecta L. (Commelinaceae), Oldenlandia corymbosa L. (Rubiaceae), Ficus exasperata Vahl (Moraceae) ont été aussi recensées. Ces plantes ont constitué l'essentiel des fourrages verts distribués aux moutons dans les mangeoires et ont été les plus abondantes sur les marchés de vente de bétail. Les herbes annuelles et vivaces ont constitué respectivement 60,8 et $30,9 \%$ des plantes utilisées dans l'alimentation des moutons sur les marchés de vente.

\section{APPETIBILITE}

La classification effectuée à partir de l'abondance des espèces présentes dans les mangeoires et l'analyse des résultats des enquêtes, montrent que plus de $70 \%$ des espèces végétales recensées dans les mangeoires ont été appétées par les moutons. Le tableau dynamique croisé obtenu, à partir des informations recueillies, montre que $23 \%$ parmi ces espèces, ont été très appétées, $39 \%$ moyennement appétées et $11 \%$ peu appétées. En outre, il a été observé que les espèces tels que Panicum maximum, qui ont été plus abondante sur les marchés d'Attécoubé et d'Abobo, et Chloris pilosa sur le marché de Port Bouët, ont été rapidement ingérées lorsque les bottes ont été servies dans les mangeoires. Ces deux espèces ont pu être considérées comme très appétées.

\section{DISCUSSION}

L'indisponibilité d'études antérieures sur l'alimentation du bétail des marchés d'Abidjan ne nous a pas permis de confronter nos résultats. La présente étude est donc pionnière dans l'analyse de l'alimentation du bétail des marchés d'Abidjan.

L'affouragement en vert a semblé être très apprécié par les vendeurs de bétail qui ont estimé que c'est le meilleur type d'alimentation pour la croissance et la prise de poids des animaux. Cependant, celui ci reste encore très dépendant de l'état de la ressource fourragère et de certaines contraintes rencontrées. Ces contraintes sont dues aux difficultés liées au transport des fourrages et aussi à l'écoulement de ceux-ci.

La complémentation en sous-produits alimentaires, apportés aux animaux, par $75 \%$ des vendeurs de bétail a permis de varier l'alimentation et a constitué également une 
source d'énergie, en complément de celle fournie par le fourrage, grâce à une teneur assez élevée en matière sèche (Boudet, 1984).

La distribution d'aliments dans les mangeoires, a été considéré comme l'unique mode d'alimentation pratiqué par les vendeurs sur les marchés de vente de bétail. Cette disposition a constitué, non seulement un moyen pour éviter la divagation des animaux, à travers les rues de la capitale, mais aussi, une solution contre les accidents de circulation, les disparitions d'animaux et l'ingestion de corps étrangers, issus de déchets domestiques principalement le plastique (Killanga et al., 1997 ; Seibou, 1996). Ce mode d'alimentation a été observé à Maroua au Cameroun (Thys et Ekembe, 1992) et à Brazzaville (Mfoukou-Ntsakala, 2000) au Congo. Cette technique permet aux vendeurs de bétail d'exploiter, au mieux, les ressources pastorales non accessibles au bétail (Klein, 2003).

Les jachères, les bas-fonds, les abords des routes, en tant que lieux d'approvisionnement, constituent des banques floristiques d'alimentation des ovins présents sur les marchés de la ville d'Abidjan, vu l'absence d'autres sources d'approvisionnement en fourrage. Ces espaces abritent des végétations qui comprennent, en effet, entre 25 et $50 \%$ d'espèces fourragères, et constituent, en général des réserves d'alimentation pour les animaux (bovins, ovins et caprins locaux ou transhumants) (Jouve, 1991 ; Richard et al., 1991 ; Hiernaux et al., 1998).

En plus d'être des réserves d'alimentation, ces zones procurent du travail à ceux qui les exploitent, leur permettant de faire face à leurs besoins quotidiens et de polier au manque d'emplois. Cette activité procure des revenus financiers se situant entre 875 et 3350 FCFA, en moyenne par jour, par exploitant. Le revenu financier moyen d'un exploitant par mois en période de pénurie d'animaux sur le marché de bétail est en moyenne de 26250 FCFA, proche du SMIG qui est de 35000 FCFA et qui peut monter jusqu'à 100400 FCFA en période d'abondance d'animaux sur le marché de vente.

Ces zones de prélèvement sont menacées de disparition par l'extension urbaine, l'accroissement démographique et le développement des infrastructures urbaines. Cette disparition entraîne une baisse, jusqu'à un manque de fourrages, vu l'absence d'autres sources d'approvisionnement à des coûts d'accessibilité acceptables. Le manque de fourrage naturel conduira les vendeurs de bétail à approvisionner leurs animaux en des produits autres que les fourrages verts, donc à rechercher des moyens financiers suffisants pour l'achat et l'entretien du bétail (Mfoukou-Ntsakala, 2000).

Les trois groupes de plantes fourragères observés sur les marchés de la ville d'Abidjan montrent en outre, une grande diversité de ressources fourragères. Les plantes annuelles ont été les plus représentatives et ont constitué l'essentiel du spectre fourrager les plus appétés et ont présenté de meilleurs résultats pour la croissance pondérale des animaux. Ces résultats corroborent ceux de (Kiéma, 1992) et (Ouédraogo, 1993), qui ont montré, au Burkina Faso que les plantes annuelles sont des espèces fourragères de très bonne qualité et mieux appétées que les plantes vivaces.

\section{CONCLUSION}

L'étude a permis de connaître les plantes fourragères consommées par les ovins sur les marchés de vente, les lieux de prélèvement de celles-ci et les contraintes liées à l'activité d'approvisionnement des marchés. Elle a révélé que l'alimentation des ovins se fait principalement à base de fourrage vert, qui permet leur maintient et leur mise en embouche. Les plantes fourragères rencontrées sur les marchés de bétail sont, pour l'essentiel, des graminées et des légumineuses. On rencontre également d'autres espèces appartenant à d'autres familles, qui rentre dans l'alimentation de ces ovins. Ces fourrages sont récoltés dans les jachères et aux abords des routes. Le mode d'alimentation pratiqué est celui de la distribution d'aliment, vu le manque d'espace approprié à une pratique pastorale de divagation des animaux en ville. La création d'espaces fourragères devéloppent la vulgarisation et la commercialisation des fourrages s'avèrent nécessaire à une modernisation de l'élevage. Le secteur de l'exploitation des ressources fourragères naturelles mérite d'être encouragé et amélioré sur le plan organisationnel, technique et financier. Cela contribuerait au développement du secteur de l'élevage dans la ville d'Abidjan et à la création d'emplois. 


\section{REFERENCES}

Anonyme. 1999. « L'agriculture ivoirienne à l'aube du XXI ${ }^{\mathrm{e}}$ siècle», Ministère d'Etat, Ministère de l'Agriculture et des Ressources Animales, Abidjan, 312 p. Publication du SARA.

Anonyme. 2001. «Aperçu de l'agriculture ivoirienne à travers les données de la base de sondage du recensement national de l'agriculture 2001, issue du RGPH98» projet GCP/IVC/025/EC - recensement national de l'agriculture - Ministère de l'Agriculture et du Développement Rural ; FAO ; UE ; juil. $2002 ; 17$ p.

Boudet G. 1984. Manuel sur les pâturages tropicaux et les cultures fourragères, $4^{\mathrm{e}}$ édition révisée, IEMVT, Paris, $266 \mathrm{p}$.

Church D. C. 1979. Taste, appetite and regulation of energy balance and control of food intake. Part. I. Appetite, taste and palatability. In : D.C. Church (Eds.), Digestive physiology and nutrition of ruminants, pp. $281-290$. Oxford Press.

Daget $P$ et J. Poissonet. 1990. Notion de valeur pastorale. Institut de Botanique. Montpellier. R. 3, pp 5 - 8

Hiernaux P., Fernandez-Rivera S., Schlecht E., Turner M. D. and T. O. Williams. 1998. Livestock-mediated nutrient transfers in Sahelian ecosystems. In : Renard G., Neef A., Becker K., von Oppen M. (Eds., Soil fertility management in West African land use systems. Weikersheim, Germany, Margraf Verlag, pp. 339-347.

Jouve P. M. 1991. Usages et fonctions de la jachère en Afrique de l'Ouest et au Maghreb. In : Floret C., Serpantié G. (Eds.). La jachère en Afrique de l'Ouest. Paris, France, Orstom, pp 55 - 66. (Colloques et séminaires)

Kiéma S. 1992. Utilisation pastorale des jachères dans la région de Bondoukuy, zone soudanienne du Burkina Faso. Mémoire DESS, Gestion des systèmes agrosylvopastoraux en zone tropicale, université de Paris XII, France, 89 p.

Killanga S., Ahmadou L. et G. Boumso. 1997. Conséquences d'une alimentation insuffisante sur la production animale dans la province de l'Extrême-Nord au Cameroun : ingestion de plastique par les bovins, les caprins et les ovins. In : Faye B. (Eds.). Socio-économie de l'élevage ovin périurbain (Secoville). Rapport scientifique d'avancement de l'année 1996 pour le Cameroun. Montpellier, France, Cirad-emvt, pp 11 -17. (Rapport $n^{\circ}$ 9)

Klein H. D. 2003. Gestion et utilisation des pâturages naturels et des prairies. Cirad- emvt /Université Montpellier II. Support de cours du DESS PARC 2003 - 2004. 10 p.

Koffi-Koumi M., Mamadou T. et B. M. Bakar. 2001. «L'élevage en Côte d'Ivoire : poids économique, développement et enjeu du secteur». In : ILRI (2001) "Quelles politiques pour améliorer la compétitivité des petits éleveurs dans le corridor central de l'Afrique de l'Ouest: Implications pour le commerce et l'intégration régionale». Proceedings of the workshop held in Abidjan, Côte d'Ivoire, 17 - 18 Septembre 2001, pp 58 - 63.

Matthews L. R. 1983. General introduction. In : Measurement and scaling of food preferences in dairy cows : concurrent schedule and free-access techniques. PhD Thesis, University of Waikato, New-Zealand, $236 \mathrm{p}$.

Mfoukou-Ntsakala A. 2000. Contribution à l'étude de l'élevage des petits ruminants en milieu urbain et périurbain de Brazzaville, Congo. Thèse M.Sc., Institut de médecine tropicale, Belgique, Anvers, $89 \mathrm{p}$.

Ouédraogo M. 1993. Ecologie comparée de deux espèces de graminées pérennes, Andropogon ascinodis C. B. Cl. et Schizachyrium sanguineum (Retz.), dans la région de Bondoukuy (Burkina Faso). Mémoire IDR, université d'Ouagadougou, Burkina Faso, $72 \mathrm{p}$.

Ould Soulé A. 1995. Utilisation de la flore de la Mauritanie. Cours de Postgrado Université de Nouakchott/Université de Barcelone, $48 \mathrm{p}$.

Richard D., Ahokpe B., Blanfort V. et B. Pouye. 1991. Utilisation des zones agricoles et pastorales par les ruminants en zone soudanienne (Moyenne Casamance, Sénégal). In : Gaston et coll. (Eds.). Actes $4^{\mathrm{e}}$ Congrès international des terres de parcours, Montpellier, France, avril 1991, pp. 759 - 762.

Rivière R. 1991. Manuel d'alimentation des ruminants domestiques en milieu tropical. Collection Manuels et précis d'élevage. $529 \mathrm{p}$.

Seibou B. 1996. Contribution à l'étude des corps étrangers du rumen chez le «mouton de case» dans la région de Dakar (Sénégal). Thèse Doct. vét., Dakar, Sénégal, 59 p.

Thys E. et T. Ekembe. 1992. Elevage citadin des petits ruminants à Maroua (Province de l'Extrême ; Nord Cameroun). Cah. Agric., 1, pp 249 - 255.

Vivier M., Michalet-Doreau B. et A. Grude. 1975. La conduite d'un troupeau laitier intensif en zone tropicale humide (Antilles Françaises). Nouv. Agron. Antilles-Guyane, 1, pp 307 - 321. 